\title{
Steady Groundwater Flow To Drains On A Sloping Bed: Comparison of Solutions based on Boussinesq Equation and Richards Equation.
}

\author{
${ }^{1,2}$ Cook F.J., ${ }^{1}$ J.H. Knight and ${ }^{1}$ R.A. Wooding \\ ${ }^{1}$ CSIRO Land and Water, ${ }^{2}$ CRC for Irrigation Futures, E-Mail: freeman.cook@csiro.au
}

Keywords: Groundwater; drains; sloping bed; Boussinesq equation, Richards equation; DupuitForchheimer

\section{EXTENDED ABSTRACT}

The steady groundwater flow of water to drains on a sloping bed is re-examined. Results from a number of solutions for the maximum watertable height $\left(h_{m}\right)$ for flow to drains on slopes are presented and compared with published HeleShaw model results and HYDRUS2D numerical model calculations. Numerical modelling shows that when the length of the slope $(L) \approx<50 \mathrm{~m}$ the ratio $L / h_{m}$ is affected by $L$ (Figure 1 ).

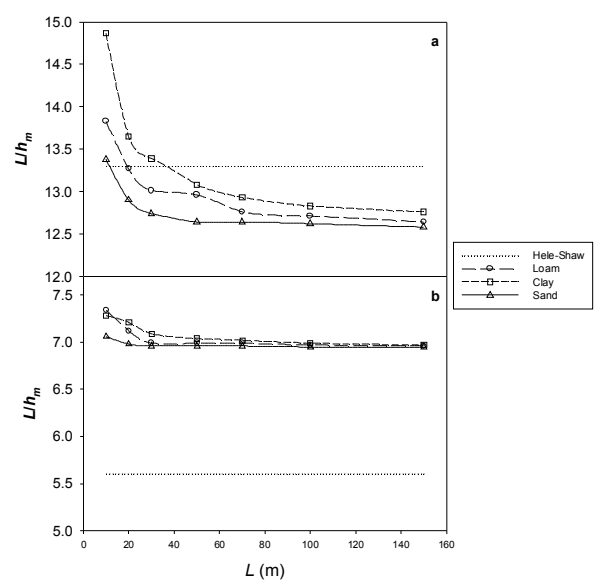

Figure 1. $L / h_{m}$ versus $L$ for the three soils used in the numerical studies and the Hele-Shaw cell model a) $p / K=0.0263, \tan \alpha=0.1$ and b) $p / K=$ $0.0927, \tan \alpha=0.7$.

Numerical simulations using Richards equation with $L=100 \mathrm{~m}$ were performed for a range of the of angle $(\alpha)$ and $p / K$ ratios ( $p$ is the steady vertical rainfall rate and $K$ is the saturated hydraulic conductivity), for three contrasting soil types. The results are similar to those of HeleShaw cell experiments when the spacing between the drains was $>50 \mathrm{~m}$. Below this spacing the $L / h_{m}$ ratio from the numerical simulations was generally greater than the Hele-Shaw and analytical results suggesting that $h_{m}$ would be over-estimated by analytical methods based on the Bossinesq equation and Dupuit-Forchheimer assumptions. This discrepancy is due to the assumptions in the analytical methods only being approximately met when $L<50 \mathrm{~m}$.

Numerical simulations of $L / h_{m}$ were then compared with analytical models based on the Boussinesq equation and Dupuit-Forchheimer assumptions. The sum of the squares of the differences $(Y)$ showed that the solution of Chapman (1980) gave the best fit (Table 1). However, if the position $\left(x_{m}\right)$ of where $h_{m}$ occurred on the slope, was compared then the solution of Towner (1975) gives the best fit to the numerical simulations.

Table 1. Sum of the squares difference $(Y)$ between $L / h_{m}$ from the numerical simulations and Hele-Shaw cell results of Guitjens and Luthin (model) and calculations of $L / h_{m}$ using Schmid and Luthin (S and L), Wooding and Chapman (W and C), Towner (T), Chapman (1980) (C1) and Chapman (2003) (C2).

\begin{tabular}{|r|r|r|}
\hline Model & $Y$, Numerical & $Y$, Hele-Shaw \\
\hline S and L & 198 & 319 \\
\hline W and C & 73 & 54 \\
\hline T & 13 & 11 \\
\hline C1 & 0.3 & 27 \\
\hline C2 & 70 & 55 \\
\hline
\end{tabular}

A computationally simple solution based on uneven drain heights gives results which are similar to the other methods when is small $\left(<3^{\circ}\right)$ and $p / K$ is large. This computationally simple solution may have application in situations with low angles and high rainfall rate relative to saturated hydraulic conductivity. These are circumstances where drains are often be required. 


\section{INTRODUCTION}

Dupuit-Forchheimer (DF) theory for groundwater flow above a horizontal plane is based on the assumption that the streamlines are horizontal and the velocity is constant in any vertical plane. This approximation clearly contravenes the boundary condition at the free surface, which will be a streamline in the absence of recharge and will have an increasing downward vertical component as recharge increases. It gives a free surface that ignores seepage surfaces, which may occur near outflow boundaries, but has been found to be an excellent approximation in the main part of the flow field. Knight (2005) has recently improved the Dupuit-Forchheimer approximation but has as yet not extended this solution to a sloping bed.

When applied without modification to flow over a sloping plane, as proposed by Schmid and Luthin (1964), DF theory also contravenes the boundary condition at the bed, with the error increasing as the bed slope increases. Wooding and Chapman (1966) sought to overcome the problem of the bed boundary condition by assuming the streamlines to be parallel to the bed, and developed a modified form that they termed 'the extended DF equation'. Their approach did not resolve the problem with the boundary condition at the free surface and its practical use was restricted by the requirement of a shape factor. Stirzaker et al. (1999) and Chapman (pers.comm, 2004) have showed that this shape factor could be found analytically. Towner (1975) used the approach taken by Schmid and Luthin (1964) but with flow parallel to the slope to derive a solution. Chapman (1980) proposed an approximation to the extended DF equation. Later Chapman and Dressler (1984) developed equations for shallow groundwater flow over a curvilinear bed, using only the assumption that the flow depths are small relative to distances along the bed. They also showed that the term in the extended DF equation, omitted by Chapman (1980) is of smaller order than the other terms.

More recently Chapman (2003, 2005) has presented an improvement to the DupuitForchheimer approximations similar to those of Knight (2005). He did not explicitly derive solutions for the maximum watertable height $\left(h_{m}\right)$, but we will do so here.

\section{SOLUTION FOR STEADY FLOW TO DRAINS ON A SLOPING PLANE}

\subsection{Schmid and Luthin Solution}

Schmid and Luthin (1964) presented the following solution for the case when all the flow exits at the lowest drain. There is one typographical error and some serious mathematical errors in their solution. Their solution for $a^{2}>4 p / K$ is:

$\ln \left(L^{2}\right)=c-\left(w_{1} / \Delta w\right) \ln w_{1}^{2}+\left(w_{2} / \Delta w\right) \ln w_{2}^{2}$

where $a=\tan \alpha, \alpha$ is the angle of the slope, $p$ is the vertical rainfall rate, $K$ is the saturated hydraulic conductivity, $L$ is the horizontal spacing between the drains (Figure 2), $c$ is a constant given by:

$$
\begin{aligned}
c= & \left(w_{1} / \Delta w\right) \ln \left[h_{m}\left(1-w_{1} \beta\right)\right]^{2} \\
& -\left(w_{2} / \Delta w\right) \ln \left[h_{m}\left(1-w_{2} \beta\right)\right]^{2}
\end{aligned}
$$

where $h_{m}$ is the maximum height of the watertable measured vertically, $\beta=K a / p$ and $w_{1}$ and $w_{2}$ are constants given by:

$$
\begin{aligned}
& w_{1}=a / 2+\left(a^{2} / 4-p / K\right)^{1 / 2} \\
& w_{2}=a / 2-\left(a^{2} / 4-p / K\right)^{1 / 2}
\end{aligned}
$$

and $\Delta w=w_{1}-w_{2}$. In Schmid and Luthin the negative sign between the first two terms in (1) is missing. This is only a typographical error, but Schmid and Luthin compound this error by solving (1) for $L$ to give:

$L=\frac{h_{m}}{w_{1} w_{2}}\left(1-w_{1} \beta\right) \exp \left[\frac{-w_{1}}{\Delta w}\right]\left(1-w_{2} \beta\right) \exp \left[\frac{w_{2}}{\Delta w}\right]$

which is incorrect. The correct solution of (1) for $L$ is:

$L=h_{m}\left|1 / w_{1}-\beta\right|^{w_{1} / \Delta w}\left(\left|1 / w_{2}-\beta\right|^{w_{2} / \Delta w}\right)^{-1}$

The values of $p / K$ versus $L / h_{m}$ presented in Figure 2 of Schmid and Luthin are correct which suggests that they used (1) to calculate their results rather than (4). It is surprising that this error has not been corrected earlier; at least we can find no reference to such a correction.

\subsection{Wooding and Chapman Solution}

Wooding and Chapman (1966) criticised the approach taken by Schmid and Luthin (1964) and solved the problem using the 'extended DupuitForchheimer' approximations, which they correctly asserted, were sounder physically. The resulting solution is similar to that of Henderson and Wooding (1964) and is:

$$
\begin{aligned}
& \lambda=4 p^{*} /\left[\left(1-p^{*}\right) a\right]^{2} \leq 1 \\
& X / A=|1-\kappa-H / X|^{(1-\kappa) / 2 \kappa} /\left(1+\kappa-H /\left.X\right|^{(1+\kappa) / 2 \kappa}\right)
\end{aligned}
$$


and for $\lambda>1$

$H / A=e^{\mu} \cos \kappa^{\prime} \mu$

$X / A=\lambda^{-1} e^{\mu}\left(\cos \kappa^{\prime} \mu+\kappa^{\prime} \sin \kappa^{\prime} \mu\right)$

where $p^{*}=p / K, X=x / L$ is the dimensionless distance parallel to the slope from the upper drain down slope, $x$ is horizontal distance down slope from the upper drain, $\kappa=(1-\lambda)^{1 / 2}, \kappa^{\prime}=(\lambda-1)^{1 / 2}, A$ is a scaling constant, $H$ is the dimensionless watertable height above the impermeable layer normal to the slope given by:

$H=2 h \cos ^{2} \alpha /\left[\left(1-p^{*}\right) L a\right]$

where $h$ is the watertable height above the impermeable layer measured vertically, $\mu$ is a parameter given by:

$\mu=1 / \kappa^{\prime} \tan ^{-1}\left([X \lambda-H] /\left(H \kappa^{\prime}\right)\right)$

A means of calculating the scaling factor $A$ was not explicitly given by Wooding and Chapman (1966) and may account for why their solution has been not been more often used prior to Towner's (1975) solution. However, $A$ can be obtained as was shown by Stirzaker et al. (1999):

$A=|1+\kappa|^{(1+\kappa) / 2 \kappa} /\left[1-\left.\kappa\right|^{(1-\kappa) / 2 \kappa}\right], \quad \lambda \leq 1$

and by (T.G. Chapman pers comm., 2003)

$A=\lambda e^{-\pi /[2(\lambda-1)]} / \kappa^{\prime}, \quad \lambda>1$

when $H(1)=0$ i.e. no seepage face at the bottom boundary. The condition that $H_{m} / X_{m}=\lambda / 2$ allows for the calculation of $h_{m}$ (the solution procedures can be obtained from the first author). Thus Wooding and Chapman's solution can be easily calculated for the whole range of $\lambda$. The physical significance of $\lambda \leq 1$ is that no water flows to the upper drain. Guitjens and Luthin (1965) noted that no flow occurred at the upper drain point in their experiments when $\lambda \leq 1$.

\subsection{Towner Solution}

Towner (1975) used a similar streamline and equipotential description to that of Wooding and Chapman (1966) but a solution method similar to Schmid and Luthin (1964). The resulting solution gives results that are identical to those of Wooding and Chapman, when $\lambda \leq 1$, but did not have an undefined shape factor, which has made the Towner solution of more practical use. This problem with the Wooding and Chapman is eliminated in this paper. For $\lambda>1$ the upper boundary condition is different between the
Towner and Wooding and Chapman solution and the results vary (Chapman, 2003).

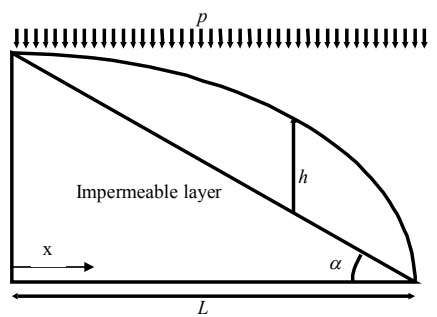

Figure 2. Schematic diagram of drain spacing on a sloping impermeable base with steady-state rainfall $p$. The slope is at an angle of $\alpha$ to the horizontal.

\subsection{Chapman Solutions}

The Chapman (1980) solution is identical to the Schmid and Luthin (1964) except that $p$ is replaced by $p \cos ^{2} \alpha$. This makes Chapman's solution practically useful. However, he found that the Towner solution gave better results when compared with the data of Guitjens and Luthin (1965).

More recently Chapman (2003) modified the Dupit-Forchheimer assumptions and derived a solution which can be solved to find the height of maximum watertable height $\left(h_{m}\right)$ as follows.

For $\lambda \leq 1$

$H_{m}=\left(\frac{2 w_{1}-\lambda}{\lambda w_{1}}\right)^{\frac{w_{1}}{w_{2}-w_{1}}} \cdot\left(\frac{2 w_{2}-\lambda}{\lambda w_{2}}\right)^{\frac{-w_{2}}{w_{2}-w_{1}}}$

and $\lambda>1$

$H_{m}=\sqrt{\lambda \exp \left[\frac{2}{\sqrt{\lambda-1}}\left\{\tan ^{-1}\left(\frac{-1}{\sqrt{\lambda-1}}\right)-\tan ^{-1}\left(\frac{\lambda-2}{2 \sqrt{\lambda-1}}\right)\right\}\right]}(13)$

where $\quad w_{1}=1+\sqrt{1-\lambda}, \quad w_{2}=1-\sqrt{1-\lambda}$, $h_{m}=H_{m}(1-p / K) L a / 2+x_{m} a p / K$ and

$x_{m}=2 H_{m} L / \lambda$.

\subsection{Solution based on Powers et al.}

The problem of flow to drains at uneven heights was studied by Powers et al. (1967) and they gave a solution using Dupuit-Forchheimer theory for the drain spacing by considering the solution to consist of separate left and right hand solutions. The left hand solution (Figure 3 ) is (their equation 82): 
$x_{m}^{2}=4(K / p)\left(h_{m}^{2}-b^{2}\right)$

and the right hand solution is (their eqn 83):

$\left(L-x_{m}\right)^{2}=4(K / p)\left(h_{m}^{2}-d^{2}\right)$

where $x_{m}$ is the distance from the drain with the lower water level to the maximum watertable height $(\mathrm{m}), d$ is the height of water in the lowest drain $(\mathrm{m})$ and $b$ is the height of water in the higher drain (m) (Figure 3). These two equations are incorrect by the constant 4 in the above equations (A.W. Warrick pers. comm.). If this constant is removed and (14) is substituted into (15) the drain spacing for uneven drain heights is given by:

$L\left(L-2 x_{m}\right)=K / p\left[\left(h_{m}^{2}-d^{2}\right)-\left(h_{m}^{2}-b^{2}\right)\right]$

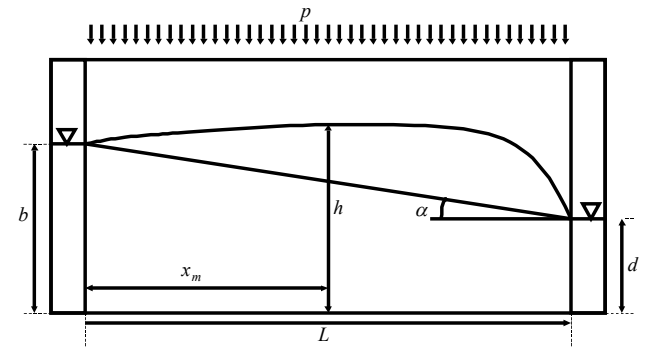

Figure 3. Schematic diagram of geometry for flow to drains at uneven drain heights. For a sloping situation, $d$ is assumed to be zero and the soil surface parallel to the slope of the line with angle $\alpha$.

Kirkham et al. (1974) suggested that the solution for uneven drain heights could be applied to drain spacing on slopes. For the situation where the angle between the horizontal and the water heights in the two drains is $\alpha$ and the height of water in the downslope drain is negligible $(d=0)$, then $b=L \tan \alpha$. Substitution for $d$ and $b$ in eqn (11) and solution for $L / h_{m}$ gives:

$$
L / h_{m}=2\left(p^{*}\right)^{1 / 2} /\left(p^{*}+\tan ^{2} \alpha\right)
$$

The simplicity of eqn (17) is attractive and may be useful when $\alpha$ is small.

\section{NUMERICAL SOLUTION FOR STEADY FLOW TO DRAINS ON A SLOPING PLANE}

Flow of water in unsaturated-saturated soil in two space dimensions is described by Richards equation (Philip, 1969):

$$
\frac{\partial \theta}{\partial t}=\nabla \cdot(k(\theta) \nabla \psi)+\frac{\partial k}{\partial z}
$$

where $\theta$ is the volumetric water content $\left(\mathrm{m}^{3} \mathrm{~m}^{-3}\right)$, $k$ is the hydraulic conductivity which is a strong function of $\theta\left(\mathrm{m} \mathrm{s}^{-1}\right)$ and $\psi$ is the matric potential of the soil $(\mathrm{m})$. This equation can be solved numerically using HYDRUS2D, a finite element model (Simunek et al., 1999) when boundary and initial conditions are specified. To simulate the steady-state flow down sloping beds the boundary conditions are shown in Figure 4. Initial conditions were chosen to be as close to the final solution as possible. The model was run until there was no change in the phreatic surface above the bottom boundary with time. The maximum head $\left(h_{m}\right)$ was obtained from the pressure along the bottom boundary. The value of $L / h_{m}$ was calculated and compared with the analytical and Hele-Shaw model results.

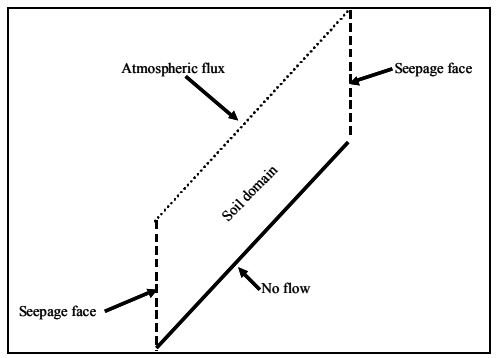

Figure 4. Schematic diagram of typical numerical domain with applied boundary conditions.

Table 2. Soil physical properties for the three soils used in the numerical study.

\begin{tabular}{|l|c|c|c|c|c|}
\hline Soil & $\begin{array}{c}\theta_{r} \\
\mathrm{~m}^{3} \mathrm{~m}^{-3}\end{array}$ & $\begin{array}{c}\theta_{\mathrm{s}} \\
\mathrm{m}^{3} \mathrm{~m}^{-3}\end{array}$ & $\begin{array}{c}\varepsilon \\
\mathrm{m}^{-1}\end{array}$ & $n$ & $\begin{array}{c}K_{s} \\
\mathrm{~ms}^{-1}\end{array}$ \\
\hline Sand & 0.045 & 0.43 & 14.5 & 2.68 & $8.25 \times 10^{-5}$ \\
\hline Loam & 0.078 & 0.43 & 3.6 & 1.56 & $2.89 \times 10^{-6}$ \\
\hline Clay & 0.068 & 0.38 & 0.8 & 1.09 & $5.56 \times 10^{-7}$ \\
\hline
\end{tabular}

Three soils, sand, loam and clay were chosen from the soil database in HYDRUS2D. The hydraulic properties; residual water content $\left(\theta_{r}\right)$, saturated water content $\left(\theta_{s}\right), \varepsilon$ and $n$ parameters in the van Genuchten (1980) moisture characteristic function and $K\left(\mathrm{~m} \mathrm{~s}^{-1}\right)$ are given in Table 2. Various sizes of the lengths of the domain from $L=10$ to $150 \mathrm{~m}$ were simulated for the lowest angle, $\tan \alpha=0.1$ and highest angle tan $\alpha=0.7$. There was little change in the value of $L / h_{m}$ obtained for $L>50 \mathrm{~m}$ (Figure 1). Hence for the rest of the simulations $L$ $=100 \mathrm{~m}$ was used. The effect of finite seepage faces and the velocity vectors not being parallel to the bed when $L<50 \mathrm{~m}$ could results in differences between the numerical and analytical models. The computational time required to reach steadystate flow can be considerable. 


\section{COMPARISONS OF SOLUTIONS WITH EXPERIMENTAL AND NUMERICAL DATA}

Guitjens and Luthin (1965) used a Hele-Shaw cell to determine values of $L / h_{m}$ for various angles and values of $p^{*}$. A Hele-Shaw cell consists of two transparent plates spaced so that the fluid flow between them is laminar and can be used to approximate fluid transport in saturated porous media. The numerical results are very similar to the Hele-Shaw cell data for all the different soils, angles and values of $p^{*}$ (Figure 5), but with the numerical simulations generally giving $L / h_{m}$ values $>$ the Hele-Shaw cell. All three soils give similar results with little scatter between them.

The Hele-Shaw cell results of Guitjens and Luthin (1965) have been used as the standard to compare analytical solutions against. We contend that the numerical solutions presented here should now be used as the standard, as the Hele-Shaw cell results incorporate problems associated with this method (see Chapman, 2005 for details).

Numerical results also indicate that for $L<50 \mathrm{~m}$ $L / h_{m}$ will be affected by $L$. Pan et al. (1997) for a $5^{\circ}$ slope and $p^{*}=0.0021$ show similar results. This is because the streamlines for flow are increasingly curved as $L$ decreases. At higher slope angles and $p^{*}$ rates the streamlines become more nearly parallel to the slope at lower values of $L$ (data not shown). Thus the assumption of horizontal (Schmidt and Luthin, 1964) or streamlines parallel to the slope in the DupuitForchheimer assumptions become less tenable as $L$ decreases. The results here would suggest that in drainage design the analytical solutions will overestimate the $h_{m}$ when $L<50 \mathrm{~m}$. This however, would not preclude their use as the results will err on the side of over design, which is often incorporated in engineering design.

Given the remarkably good comparison between the Hele-Shaw cell results and the numerical simulations shown in figure 5, the numerical results will be used as the standard for comparison with the analytical solutions. The mean of the three simulations (sand, loam, clay) for each angle, $p^{*}$ point was calculated. As a measure of difference between an analytical solution and the mean numerical value, the sum of the squares of the difference $(Y)$ was calculated:

$Y=\sum_{i=1}^{n}\left(N_{i}-M_{i}\right)^{2}$ where $i$ is an index variable associated with the particular angle and $p^{*}, n$ is the total number of data points, $N_{i}$ is either the mean numerical value or Hele-Shaw cell value of $L / h_{m}$ for $i$, and $M_{i}$ is the analytical model vale of $L / h_{m}$ for $i$. The results show that Chapman's (1980) solution gives the best fit followed by Towner's with Wooding and Chapman's, Chapman (2003) and Schmid and Luthin's (Table 1). However, if the Hele-Shaw cell data was used as the comparison then Towner's solution fits best, followed by Chapman (1980).

We recalculated all the values rather than use the published values, as the calculations that Guitjens and Luthin (1965) presented for $L / h_{m}$ using the Schmid and Luthin solution were incorrect in a few cases, particularly at the larger angles. This may have been due to the rounding errors due to the computational methods they used. The values presented here indicate that although the Schmid and Luthin solution grossly overestimates $L / h_{m}$ at large slope angles, it is not by as much as Guitjens and Luthin indicated.

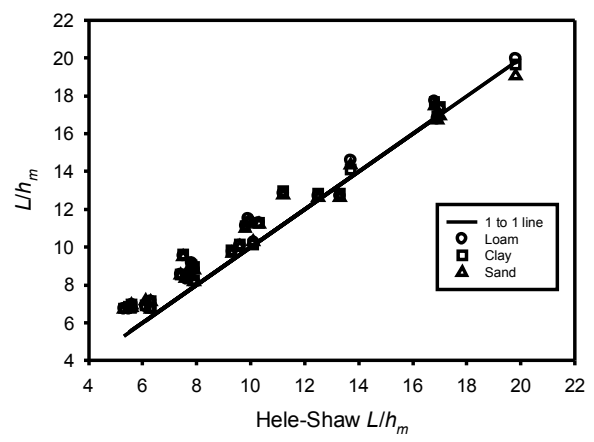

Figure 5. Comparison of $\mathrm{L} / \mathrm{h}$ from numerical study for three soils with Hele-Shaw model results. The line is a 1 to 1 line.

The sum of the squares of the differences between the the Hele-Shaw cell results and those calculated with the various analytical methods confirm that the solutions of Towner (1975) give the best fit with Guitjens and Luthin's data (Table 1). This good fit applies to the range of angles and $p^{*}$ values in Guitjens and Luthin's study (Figure 6). However, when compared with the numerical simulations both Towner's solution and the HeleShaw cell results underestimate $L / h_{m}$ at low values of $L / h_{m}$. The Wooding and Chapman (1966) solution gives identical results to those of Towner when $\lambda<1$ but diverged when $\lambda>1$ (Figure 6). This difference is worst at low slopes and high $p^{*}$. Chapman's (1980) solution fits the numerical data 
remarkablly well throughout the range of angles and $p^{*}$ values.

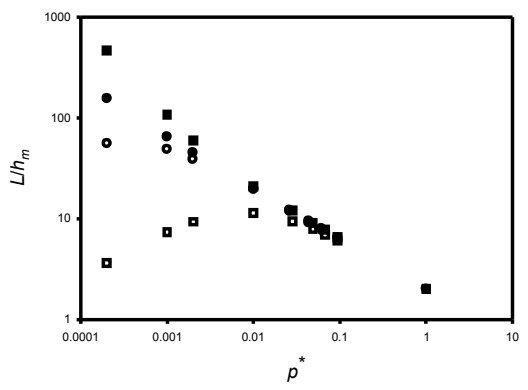

Figure 6. Relationship between $p / K$ and $L / h_{m}$ at slopes of $1^{\circ}$ and $5^{\circ}$ calculated with Chapman $(1980)(\bullet,-$ respectively) and eqn (17) $(O, \square$ respectively).

When compared with the Hele-Shaw results Chapman's (1980) and (2003) solutions gave similar sums of the squares of the deviation, but the differences occur at different ranges. The Chapman (1980) fits well when tan $\alpha<0.1$ while Chapman (2003) fits poorly in this range but fits well for the rest of the range. This fit of Chapman (1980) at low angles confirms the conclusion by Chapman and Dressler (1984) that the term in the extended DF theory omitted by Chapman (1980) in developing his approximation is of smaller order than the other terms. The numerical results would also suggest that this approximation is negligible.

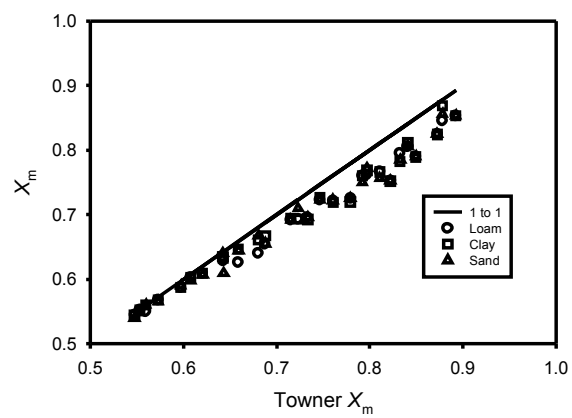

Figure 7. $X_{m}$ calculated using Towner (1975) compared with values derived from numerical simulations of 3 different soil types.

The approximate solution using eqn (17) was compared with Chapman's (1980) solution for angles of $1^{\circ}$ and $5^{\circ}$ (Figure 6). For values of $p^{*}>$ 0.03 and angles of up to $3^{\circ}$ the values of $L / h$ calculated with eqn (17) are less than $10 \%$ different from those calculated using Chapman's solution. These results suggest that for such conditions eqn (17) would provide a rapid method for calculating drain spacing.

It is also instructive to determine where along the slope the maximum watertable height $\left(X_{m}=x_{m} / L\right)$ is predicted to occur. The $X_{m}$ results from the numerical simulations, with $L=100 \mathrm{~m}$, are shown to correspond well with those of Towner (1975) (Figure 7). Towner's method seems to overestimate $X_{m}$ at high values when the slope is high, but generally gives a good estimate.

The value of $X_{m}$ was also calculated with the other analytical solutions and compared with the mean value from the three soil types at each (angle, $p$ ) data point. All of the other methods underestimate $X_{m}$ at low slope values (Figure 8). The Wooding and Chapman (1966) and Chapman (2003) gave identical values for $X_{m}$, even though they gave different values for $L / h_{m}$. The Chapman (1980) and Schmid and Luthin (1964) also gave similar results for $X_{m}$. This underestimation of $X_{m}$ will result in the maximum height of the watertable being predicted to be closer to the upslope drain.

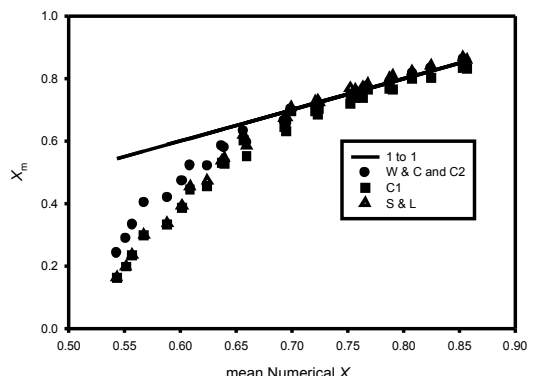

Figure 8. $X_{m}$ calculated using; Wooding and Chapman (1966) (W \& C), Chapman (2003) (C2), Chapman (1980) (C1) and Schmid and Luthin (1964) (S \& L), compared with the mean value derived from numerical simulations of 3 different soil types.

\section{CONCLUSIONS}

Numerical results of flow to drains on a slope calculated using Richards equation for three contrasting soil types produce similar results to Hele-Shaw cell experiments when the spacing between the drains was $>50 \mathrm{~m}$. Below this spacing the $L / h_{m}$ ratio was greater than the HeleShaw and analytical results suggesting that $h_{m}$ would be over-estimated by analytical methods based on the Bossinesq equation and DupuitForchheimer assumptions. This discrepancy is due to the assumptions in the analytical methods only being approximately met when $L<50 \mathrm{~m}$. 
Comparison of a number of methods for calculating the drain spacing for steady flow down a sloping plane with the numerical simulations showed that Chapman's (1980) solution gave the best fit for the maximum height of the watertable. While Towner's (1975) was best a describing where this maximum height occurred on the slope.

A method for calculating the scaling factor in Wooding and Chapman's (1966) solution for all the solution range is presented and adds to the utility of this method. Identical results compared to Towner are obtained when $\lambda<1$ and no discharge occurs at the upper drain.

A solution based on flow between two drains at unequal heights by Powers et al. (1967) contained an error, which is corrected here. This solution diverges from the solution of Chapman's at small values of $p / K$ and/or large values of $\alpha$. However, it could be used as a rapid means of calculating drain spacing at small slope angles $\left(<3^{\circ}\right)$ and high relative flow rates $\left(p^{*}>0.03\right)$.

\section{ACKNOWLEDGEMENTS}

Professor Tom Chapman is thanked for his comments and support. The senior author would like to thank his long suffering family who had to put up with the considerable time and computing power required for these simulations.

\section{REFERENCES}

Chapman, T.G. (1980), Modelling groundwater flow over sloping beds, Water Resources. Research, 16, 1114-1118.

Chapman, T.G. and R.F. Dressler (1984), Unsteady shallow groundwater flow over a curved impermeable boundary, Water Resources Research, 20, 1427-1434.

Chapman, T.G. (2003), Steady recharge-induced groundwater flow over a plane bed: nonlinear and linear solutions, International Congress on Modelling and Simulation, Townsville, July 2003.

Cahpman, T.G. (2005), Recharge-induced groundwater flow over a plane sloping bed: Solutions for steady and transient flow using physical and numerical models. Water Resources Research, 41, W07027.

Guitjens, J.C. and J.N. Luthin (1965), Viscous model study of drain spacing on sloping land and comparison with mathematical solution, Water Resources Research, 1, 523-530.
Henderson, F.M., and R.A. Wooding (1964), Overland flow and groundwater flow from a steady rainfall of finite duration, Journal of Geophysical Research, 69, 1531-1540.

Knight, J.H. (2005), Improving the DupuitForchheimer groundwater free surface approximation, Advances in Water Resources 10, 1048-1056.

Kirkham, D., S. Toksöz, and R.R. van der Ploeg (1974), Steady flow to drains and wells, In Drainage for Agriculture. Ed. J. van Schilfgaarde. 203-244.

Pan, L., A.W. Warrick, P.J. Wierenga, (1997), Downward water flow through sloping layers in the vadose zone: time-depenebce and effect of slop length. Journal of Hydrology, 199, 36-52.

Philip, J.R. (1969), The theory of infiltration, Hydrosciences, 5, 215-296.

Powers, W.L., D. Kirkham, and G. Snowden (1967), Seepage of steady rainfall through soil into ditches of unequal level heights, Soil Science Society of America Proceedings, 31, 301-312.

Schmid, P., and J.N. Luthin (1964), The drainage of sloping lands, Journal of Geophysical Research, 69, 1525-1529.

Simunek, J., M. Sejna and M.Th. van Genuchten, (1999), HYDRUS-2D / MESHGEN-2D:

Simulating water flow and solute transport in two-dimensional variably saturated media. International Groundwater Modeling Centre, Colorado School of Mines, Golden, Colorado, 1999.

Stirzaker R.J., F.J. Cook, and J.H. Knight, (1999). Where to plant trees on cropping land for control of dryland salinity: some approximate solutions, Agricultural Water Management, 35, 115-133.

Towner, G.D. (1975), Drainage of groundwater resting on a sloping bed with uniform rainfall. Water Resources Research, 11, 144-147.

van Genuchten, M. Th., (1980), A closed-form equation for predicting the hydraulic conductivity of unsaturated soils, Soil Science Society of America Journal, 44, 892-898.

Wooding, R.A. and T.G. Chapman (1966), Groundwater flow over a sloping impermeable layer. 1. Application of the Dupuit-Forchheimer assumption. Journal of Geophysical Research, 71, 2895-2902. 